\title{
Influence of Polarization Processes on the Morphology and Photoluminescence Properties of $\mathrm{PP} / \mathrm{TiO}_{2}$ Polymer Nanocomposites
}

\author{
M.A. Ramazanov*, F.V. Hajiyeva, A.M. Maharramov, A.B. Ahmadova, \\ U.A. Hasanova, A.M. Rahimli and H.A. ShiRinova \\ Baku State University, Zahid Khalilov Str. 23, AZ 1148, Baku, Azerbaijan
}

(Received January 22, 2017; in final form April 14, 2017)

The paper reports of synthesis and investigation of polymeric nanocomposites based on isotactic polypropylene and titanium dioxide nanoparticles $\mathrm{PP}+\mathrm{TiO}_{2}(\mathrm{PPT})$. The structure of the PPT nanocomposites was studied by scanning electron and atomic force microscopy. There was also studied the influence of the polarization process by corona discharge on the structure and photoluminescence properties of PPT nanocomposites. It was found that intensity of the photoluminescence after the polarization increases, and this depends on the concentration of titan dioxide nanoparticles in the polymer matrix. It was shown that rms roughness for non-polarized sample compositions is $60-100 \mathrm{~nm}$, whereas for polarized samples after the corona discharge polarization, makes 20-40 nm, i.e. there takes place grinding of the structural elements. It is supposed that, in the composite there forms sufficiently high internal local field, due to the boundary charges, so under the influence of this field there were excited additional luminescent centers, and as a result, after the polarization there was observed the increase of luminescence intensity.

DOI: 10.12693/APhysPolA.131.1540

PACS/topics: photoluminescence, corona discharge, nanocomposites, polypropylene, titanium dioxide

\section{Introduction}

Currently, organic-inorganic polymer nanocomposites have attracted attention due to its unique properties: mechanical, magnetic, optical, electronic, chemical resistance, resistance to UV-radiation. It is known that the combination of components with different chemical structures can lead to the formation of a material with improved characteristics compared with properties of individual components. Impact of filler on the properties of composites depends on the concentration, particle size, aggregation degree and character of its interaction with the polymer [1]. Nanocrystalline $\mathrm{TiO}_{2}$ is a wide-gap semiconductor, and has a number of advantages (good photocatalytic activity, high chemical and thermal stability, non-toxicity, and low cost). Use of composites containing $\mathrm{TiO}_{2}$ nanoparticles is determined mostly by their photocatalytic properties (creation of solar cells, UV filters, gas sensors).

$\mathrm{TiO}_{2}$ has been widely used for experiments related to solar energy conversion. Furthermore, $\mathrm{TiO}_{2}$ reveals photoluminescence at ambient temperature or below under various conditions [2-4]. Titanium dioxide also shows electroluminescence with a spectral distribution below the band gap, i.e., luminescence also occurs from levels within the bandgap. The quantum efficiency in these experiments was very low, suggesting efficient nonradiative recombination. As far as we know there are very

*corresponding author; e-mail: mamed_r50@mail.ru few studies about the temperature dependence of powdered $\mathrm{TiO}_{2}$ luminescence at temperatures above $300 \mathrm{~K}$. In Ref. [4] Hashimoto et al. described that the luminescence intensity increased steadily with decreasing temperature and became almost constant below $77 \mathrm{~K}$. They also observed an excitation spectrum that extended up to $600 \mathrm{~nm}$ and corresponds to the luminescence spectrum from the surface states.

In this paper, we report of the synthesis of polymer nanocomposite on the basis of polypropylene and titania nanoparticles PPT and study the effect of corona discharge on the physical structure and photoluminescence properties of obtained nanocomposite.

\section{Experimental part}

\subsection{Synthesis of PPT nanocomposites}

The isotactic polypropylene powder used as polymer matrix (PP brand Sigma Aldrich Pcode 1,001,326,963) nanoparticles of titania - as a filler $\left(\mathrm{TiO}_{2}\right.$ rutile, $99.5 \%$, product \#7920DL, lot \#7920-091012, SkySpringNanomaterials, Inc., Houston). Polymer nanocomposites $\mathrm{PP}+\mathrm{TiO}_{2}(\mathrm{PPT})$ were prepared as follows: isotactic polypropylene powder at a temperature of $120^{\circ} \mathrm{C}$ was dissolved in an organic solvent, toluene. Then, to the polymer solution there was added nanopowder of $\mathrm{TiO}_{2}$ at a temperature of $393 \mathrm{~K}$. The mixture was transferred to a Petri dish and dried in a vacuum oven during $24 \mathrm{~h}$. Preparation of nanocomposite film samples was made by hot pressing at the melting temperature of polypropylene and a pressure of $15 \mathrm{MPa}$ for $10 \mathrm{~min}$ and then cooling under pressure at room temperature. The thicknesses of the films vary within $100 \mu \mathrm{m}$. 


\subsection{Method of polarization of polymer nanocomposites}

Polarization nanocomposites were performed using a corona discharge. Corona discharge was created via needle-plane electrodes. The needle diameter is about $0.3 \mathrm{~mm}$, and the distance between the needle and the film sample $1 \mathrm{~cm}$. The charging voltage $U_{k} \approx 6-9 \mathrm{kV}$, the charging time is $5-10 \mathrm{~min}$. The sample thickness is $100 \mu \mathrm{m}$. Before starting the experiment samples were thoroughly degreased. Thereafter, they were placed on a grounded electrode and at a voltage of $6 \mathrm{kV}$ and were charged by a negative corona discharge by means of metal needles system arranged vertically at a distance of $6 \mathrm{~mm}$ from the sample surface.

\subsection{AFM analysis}

The morphology of the nanocomposites was studied using atomic force microscopy Integra Prima (NT-MDT, Zelenograd). For the scan used special silicon cantilevers fabricated by plasma etching method with a radius of curvature of the needle $20 \mathrm{~nm}$ and the resonance frequency of $1-5 \mathrm{~Hz}$. Scan site was $2 \times 2 \mu \mathrm{m}^{2}$. The measurements were performed in tapping microscopy mode in air, the changes in the oscillation amplitude of the cantilever needle that defines the surface topography was recorded. The scanning rate and the number of scanned lines in the image are respectively $256 \mathrm{~Hz}$ and 1969 .

\subsection{SEM analysis}

Distribution of titanium oxide nanoparticles in the polymer matrix studied by scanning electron microscopy (JEOL JSM - 7600F) was conducted at scanning SEI mode at an accelerating voltage of $15 \mathrm{keV}$ working distance of $4.5 \mathrm{~mm}$. To eliminate the excess charge from the surface of polymeric nanocomposite was covered by platinum layer of $10 \mathrm{~nm}$ thick.

\subsection{Photoluminescent analysis of nanocomposites}

Photoluminescent properties of polymer nanocomposites film were studied by spectrofluorometer Varian Cary Eclipse in the wavelength range $200-900 \mathrm{~nm}$.

\section{Results and discussion}

Figure 1 shows the diffraction patterns of titanium oxide nanoparticles. XRD diffraction patterns show that the main peaks of 27.506 (110), 36.040 (101), 41.185 (111), 44.140 (210), 54.231 (211), 56.781 (220), 62.258 (002), and 68.996 (301) belong to the rutile phase of titanium dioxide nanoparticles. In this pattern, all lines can be indexed using the ICDD no. 00-001-1292 corresponding to rutile.

Figure 2 shows SEM images of polymer nanocomposites based on PPT. As seen from Fig. 2 there was observed the homogeneous distribution of titanium dioxide nanoparticles in the polymer matrix. It was also found that with increase of nanoparticle content in the polymer matrix there was observed a significant coagulation

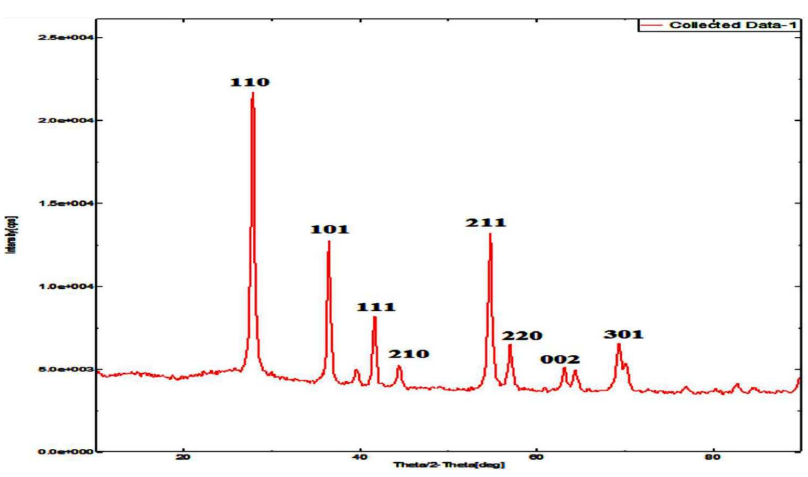

Fig. 1. XRD diffraction pattern of $\mathrm{TiO}_{2}$ nanoparticles.
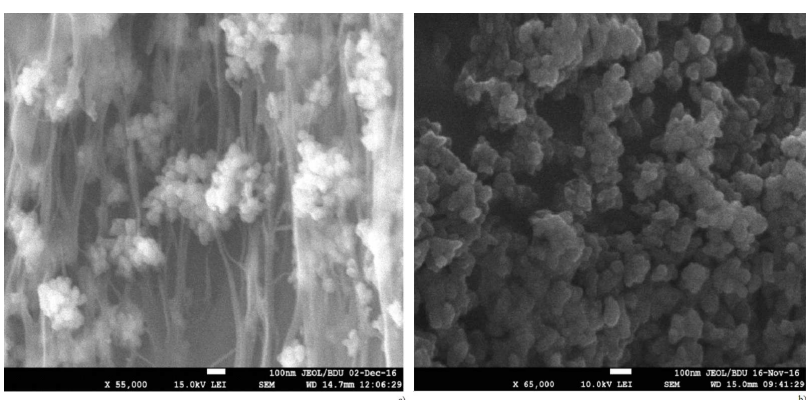

Fig. 2. SEM images of nanocomposites based on PPT:

(a) PPT5, (b) PPT10.

of the $\mathrm{TiO}_{2}$ nanoparticles. Thus, at the $5 \% \mathrm{TiO}_{2}$ content in the polymer (PPT5) the size of nanoparticle is about 10-30 nm, and at 10\% (PPT10) - 50-60 nm. Figures 3 and 4 show the mapping of the elements and EDS spectrum correspondingly for PPT nanocomposite. As seen from Figs. 3 and 4 nanocomposite contain titanium dioxide. The appearing of platinum in the spectra is due to coating by a thin layer of platinum the surface of the PPT sample in order to avoid the charging of polymer film.
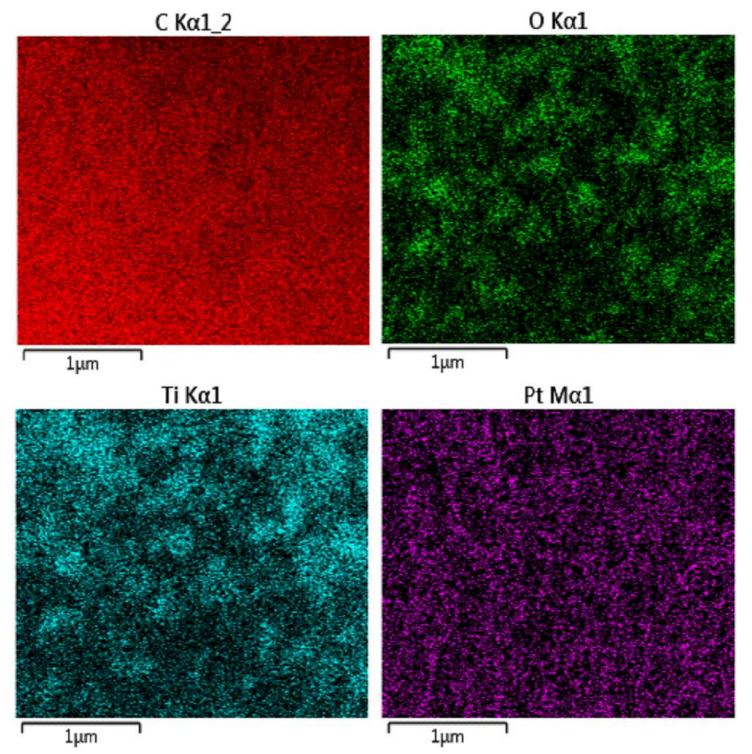

Fig. 3. Mapping of the elements for a nanocomposite based on PPT. 


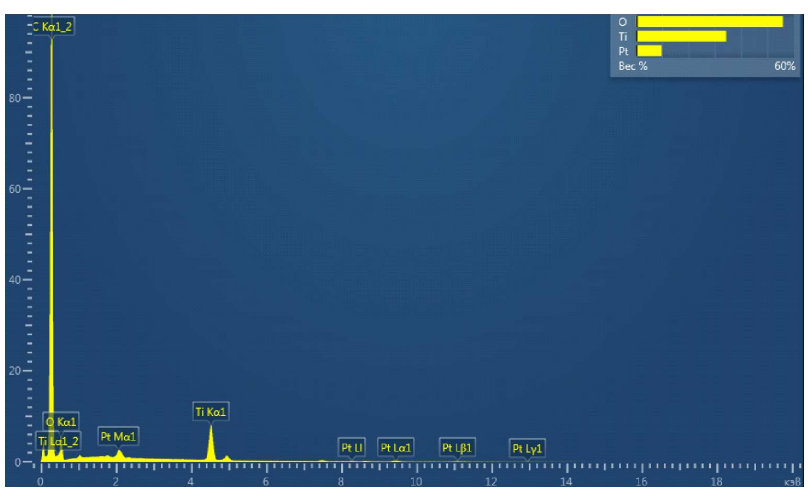

Fig. 4. Energy dispersive spectrum (EDS) for nanocomposite based on PPT.

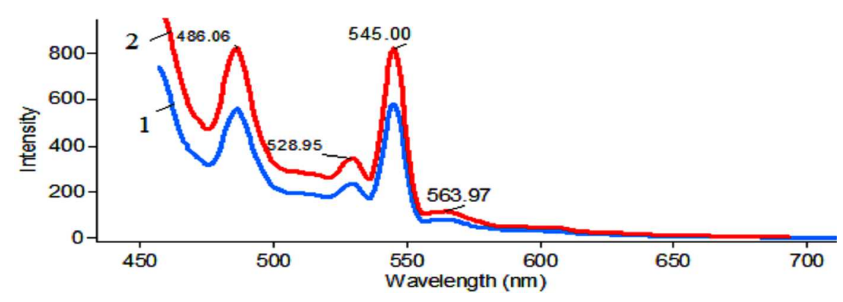

Fig. 5. Photoluminescence spectra nanocomposite PPT5 before (1) and after (2) the polarization by corona discharge.

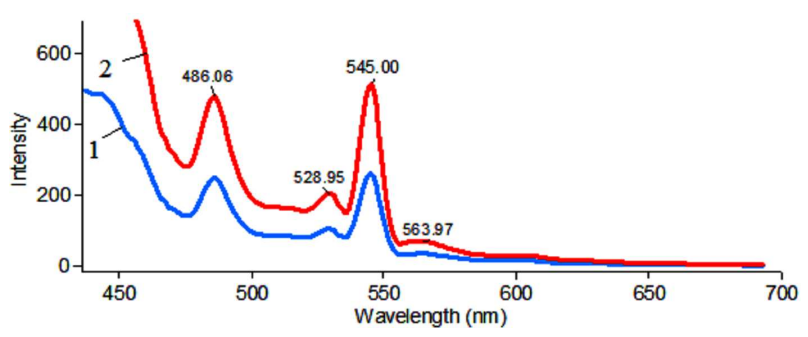

Fig. 6. Photoluminescence spectra of nanocomposite PPT10 before (1) and after (2) the polarization by corona discharge.

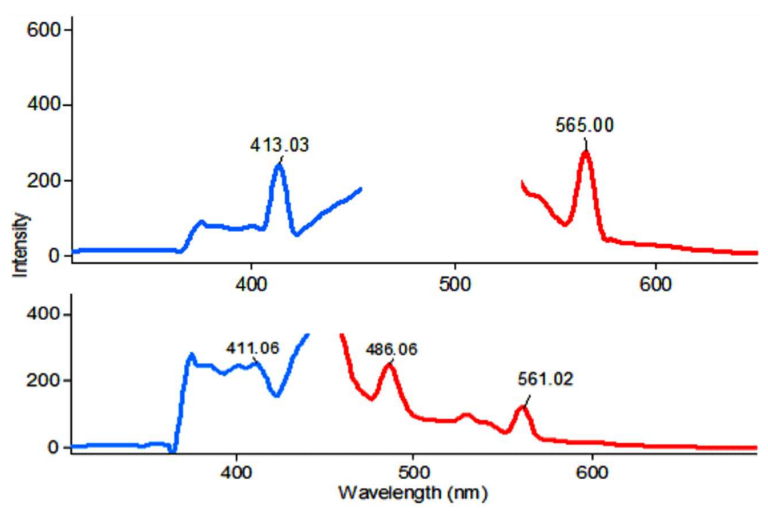

Fig. 7. Photoluminescence excitation and emission spectra of PPT nanocomposites at different excitation wavelengths.
The photoluminescence spectra (PL) of PPT nanocomposites were studied at 5\% (PPT5)and $10 \% \mathrm{TiO}_{2}$ (PPT10)concentrations by volume of contents in the wavelength range of $450-700 \mathrm{~nm}$ with excitation at $400 \mathrm{~nm}$ wavelength. It was found that the photoluminescence intensity of PPT nanocomposite varies with the extremum, the PL intensity gets its maximum value for PPT5 nanocomposite. Figures 5 and 6 show the luminescence spectra of the nanocomposite PPT5 and PPT10 before (1) and after (2) of polarization by corona discharge. It is found that the peaks in the luminescence spectra observed at wavelengths of $486,528,545$, and $563 \mathrm{~nm}$. It was determined that the two peaks at wavelengths of $486 \mathrm{~nm}$ and $563 \mathrm{~nm}$ are photoluminescent peaks belonging to titanium dioxide (Fig. 7). From Figures 5 and 6 it can be seen that for PPT5 nanocomposite the photoluminescence intensity is higher than for the composite PPT10. In our opinion it can be explained by agglomeration of $\mathrm{TiO}_{2}$ nanoparticles with an increase of the content of $\mathrm{TiO}_{2}$ in polypropylene, resulting in increase of the nanoparticles' size, that in turn lead to a decrease in the specific surface of the luminescent nanoparticles. Thus it is assumed that the change in PL amplitude with extremum associated with a reduced of specific surface area of $\mathrm{TiO}_{2}$ at high concentrations $\mathrm{TiO}_{2}$ nanoparticles.

Also photoluminescence spectra of PPT nanocomposites were investigated before and after the polarization by the corona discharge. It was found that the photoluminescence intensity increases after polarization, and an increase of luminescence intensity depends on the concentration. In previous studies [5-7] it was shown that in the interfacial layer of nanocomposites there were large number of cross-border charges generated as a result of the polarization of the polymer nanocomposites by different physical effects (electro-thermo-polarization, polarization by corona discharge). These charges in turn create high internal local field and under the influence of this field in polymer nanocomposites excited additional fluorescent centers of titanium dioxide nanoparticles. As a result, after the polarization by corona discharge increased luminescence intensity of nanocomposites based on PPT.

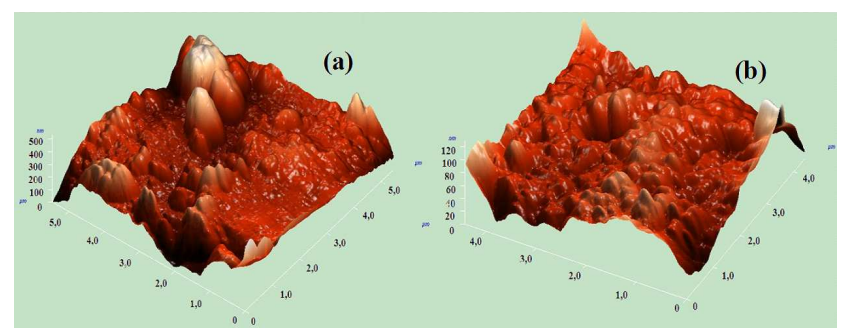

Fig. 8. AFM images of polymer nanocomposites based on PPT to (a) and after (b) polarization.

The surface of PPT5 nanocomposites was studied by atomic force microscopy method before and after the polarization under the influence of corona discharge $[8,9]$. 
Figure 8 shows 3D images of PPT nanocomposite before and after the polarization by the corona discharge. AFM topography study of the sample compositions PPT shows that the relief of the composition samples varies strongly after the polarization under the influence of corona discharge. It can be seen that on the surface of the composition samples after polarization the structural changes occur.

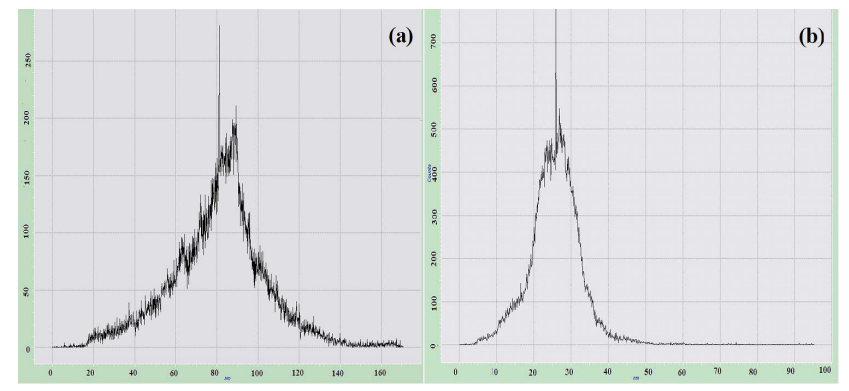

Fig. 9. Histogram of rms roughness of polymer-based nanocomposites PPT before (a) and after (b) polarization.

Figure 9 shows a histogram of pixel values, and the rms roughness of the surface compositions of PPT. Histogram heterogeneity of the surface reveals that after the polarization relief composition is relatively non-smooth. It is also shown that the rms surface roughness of samples for non-polarized samples is $80-120 \mathrm{~nm}$ and for the polarized sample is $40-60 \mathrm{~nm}$, i.e. after polarization there occurs the grinding of structural elements of nanocomposites.

\section{Conclusion}

In the paper we report of synthesis and investigation of polymeric nanocomposites based on isotactic polypropylene and titanium dioxide nanoparticles. The structure and morphology of the nanocomposites was studied by scanning electron (SEM) and atomic force microscopy (AFM). It was also studied the influence of the polarization process by corona discharge on the structure and photoluminescence properties of PPT nanocomposites.
The intensity of the photoluminescence after the polarization increases, and this depends on the concentration of titanium dioxide nanoparticles in the polymer matrix. It was shown that rms roughness for non-polarized sample compositions is $60-100 \mathrm{~nm}$, whereas for polarized samples after the polarization under the influence of corona discharge, it makes $20-40 \mathrm{~nm}$, i.e. there takes place grinding of the structural elements. It is supposed that, in the PPT composite sufficiently high internal local field forms, due to the boundary charges, so under the influence of this field there were excited additional luminescent centers, and as a result, after the polarization there was observed the increase of luminescence intensity.

\section{References}

[1] A.D. Pomogaylo, V.N. Kestelman, Metallopolymer Nanocomposites, Springer Series in Materials Science, Vol. 81, 2005, p. 671.

[2] L. Forss, M. Schubnell, Appl. Phys. B 56, 363 (1993).

[3] S.K. Poznyak, V.V. Sviridov, A. Kulak, M.P. Samtsov, J. Electroanalyt. Chem. 340, 73 (1992).

[4] K. Hashimoto, M. Hiramoto, T. Sakata, in: Proc. Symp. on Photoelectrochemistry and Electrosynthesis on Semiconducting Materials, 88-14, The Electrochemical Society, Pennington 1988, p. 395.

[5] M.A. Ramazanov, F.V. Hajiyeva, A.M. Maharramov, J. Ferroelectr. 493, 103 (2016).

[6] M.A. Ramazanov, A.S. Quseynova, Optoelectron. Adv. Mater., Rapid Commun. 7, 789 (2013).

[7] M.A. Ramazanov, A.S. Huseynova, F.V. Hajiyeva, J. Ovon. Res. 11, 35 (2015).

[8] M.A. Ramazanov, F.V. Hajiyeva, A.M. Maharramov, U.A. Hasanova, J. Ferroelectr. 507, 121 (2017).

[9] M.A. Ramazanov, A.S. Guseinova, J. Surf. Eng. Appl. Electrochem. 49, 97(2013). 